BULL. AUSTRAL. MATH. SOC.

VOL. 34 (1986) 157-159.

\title{
SOLVABILITY OF NON SELF-ADJOINT \\ AND HIGHER ORDER DIFFERENTIAL \\ EQUATIONS WITH JUMPING NONLINEARITIES
}

\section{Peter J, Pope}

This thesis is concerned with problems of the type

$$
L u-g(u)=f(x)
$$

We assume that the nonlinearity in (1) can be written as

$g(u)=\mu u^{+}+v u^{-}+\psi(u)$ where $\mu, v \in \mathbb{R}, \mu=\lim _{u \rightarrow \infty} u^{-1} g(u)$,

$v=\lim _{u \rightarrow-\infty} u^{-1} g(u)$. In the Ambrosetti-Prodi result the nonlinearity

'jumps' over the first eigenvalue of the linear problem, that is, $\nu<\lambda_{1}<\mu \quad$ (see [1]).

Knowledge of the set $A_{0}=\left\{(\mu, \nu) \in \mathbb{R}^{2}\right.$ : there exists a nontrivial solution of $\left.L u=\mu u^{+}+v u^{-}\right\}$is important if one wishes to apply homotopy invariant index methods to establish the existence of solutions. We obtain results for the set $A_{0}$ for a wide class of operators $L$.

$A_{0}$ is in general difficult to calculate but one problem for which results are known is the second-order self-adjoint problem:

Received 28 January 1986. Thesis submitted to University of New England, January 1985. Degree approved October 1985. Supervisor: Professor E.N. Dancer.

Copyright Clearance Centre, Inc. Serial-fee code: 0004-9727/86 $\$ A 2.00+0.00$. 
158

$$
\begin{aligned}
& -u^{\prime \prime}(x)-\mu u^{+}(x)-v u^{-}(x)=0, \quad x \in(0, \pi) \\
& u(0)=u(\pi)=0
\end{aligned}
$$

Knowledge of $A_{0}$ for this problern has been suggestive of a number of more general results. (See [2].)

We calculate the set $A_{0}$ explicitly for two problems:

(i) The second-order non self-adjoint problem,

$$
\begin{gathered}
-u^{\prime \prime}(x)-u u^{+}(x)-\nu u^{-}(x)=0, \quad x \in(0, \pi) \\
u(0)+u(\pi)=0, u^{\prime}(\pi)=0
\end{gathered}
$$

(ii) The fourth-order self-adjoint problem suggested by Fučik in [4],

$$
\begin{aligned}
& -u^{i v}(x)-\mu u^{+}(x)-\nu u^{-}(x)=0, x \in(0, \pi) \\
& u(0)=u(\pi)=u^{\prime \prime}(0)+u^{\prime \prime}(\pi)=0
\end{aligned}
$$

These results compare interestingly with the known case and in the secondorder case we obtain a counter-example to a potential generalisation of a classic theorem of Dolph.

Dancer's results in [2] for the complementary set, $\mathbb{R}^{2} \backslash A_{0}$, are extended for higher-order and non self-adjoint problems. Here the use of the Maximum Principle is foregone and the theory of Positive Operators used instead.

More general theorems are obtained concerning the local behaviour of the set $A_{0}$ near $\left(\lambda_{k}, \lambda_{k}\right)$ both when $\lambda_{k}$ is a sirple eigenvalue and when $\lambda_{k}$ is an eigenvalue of higher multiplicity for the corresponding linear problem. The difficulty here is the non-smoothness of the nonlinearity in (1). (It is not even $C^{1}$ ). A modification of the Implicit Function Theorem is used.

Finally, in the case when the linearisation has one-dimensional kernel, we provide a negative answer to a question posed by Dancer in [3]: Does $A_{0}$ contain an open set? 


\section{References}

[1] A. Ambrosetti and G. Prodi, "On the inversion of some differentiable mappings with singularities between Banach Spaces", Ann. Mat. Pure App z. 93 (1972), 245-254.

[2] E.N. Dancer, "On the Dirichlet problem for weakly nonlinear elliptic partial differential equations", Proc. Royal Soc. Edinburgh $76 \mathrm{~A},(1977), 283-300$.

[3] E.N. Dancer, "Solvability of some asymptotically homogeneous elliptic problems", Proc. Centre for Mathematical Analysis, A.N.U. Vol. 1. Miniconference on Partial Differential Equations, Canberra, A.N.U. Press (1982) 106-111.

[4] S. Fucik, Solvability of nonlinear equations and boundary value problems, (D. Reidel Publishing Company, North-Holland).

Department of Econometrics,

University of New England,

Armidale, 2351

New South wales. 